\section{BRAZIULIAN JOURNAL}

OF MEDICAL AND BIOLOGICAL RESHARCH

www.bjournal.com.br
ISSN 0100-879X

Volume 43 (10) 914-1009 October 2010

BIOMEDICAL SCIENCES

AND

CLINICAL INVESTIGATION

Braz J Med Biol Res, October 2010, Volume 43(10) 982-988

doi: 10.1590/S0100-879X2010007500101

Reproducibility of heart rate variability parameters measured in healthy subjects at rest and after a postural change maneuver

E.M. Dantas, C.P. Gonçalves, A.B.T. Silva, S.L. Rodrigues, M.S. Ramos, R.V. Andreão, E.B. Pimentel, W. Lunz and J.G. Mill

The Brazilian Journal of Medical and Biological Research is partially financed by
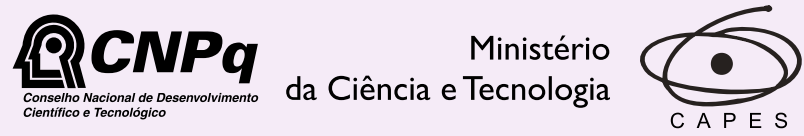

Ministério da Educação

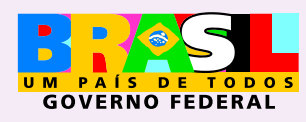

DTAPESP

Institutional Sponsors
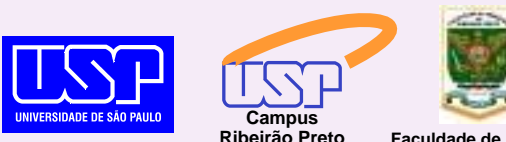

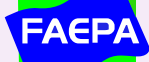

Ribeirão Preto $\oplus$ SHIMADZU

GE Healthcare
Hotsite of proteomics metabolomics developped by:

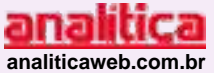

Thermo
SCIENTIFIC 


\title{
Reproducibility of heart rate variability parameters measured in healthy subjects at rest and after a postural change maneuver
}

\author{
E.M. Dantas ${ }^{1}$, C.P. Gonçalves ${ }^{1}$, A.B.T. Silva ${ }^{1}$, S.L. Rodrigues ${ }^{1}$, M.S. Ramos ${ }^{2}$, \\ R.V. Andreão ${ }^{2}$, E.B. Pimentel ${ }^{1}$, W. Lunz ${ }^{1}$ and J.G. Mill ${ }^{1}$ \\ ${ }^{1}$ Departamento de Ciências Fisiológicas, Universidade Federal do Espírito Santo, Vitória, ES, Brasil \\ ${ }^{2}$ Centro Federal de Educação Tecnológica do Espírito Santo, Vitória, ES, Brasil
}

\begin{abstract}
Heart rate variability (HRV) provides important information about cardiac autonomic modulation. Since it is a noninvasive and inexpensive method, HRV has been used to evaluate several parameters of cardiovascular health. However, the internal reproducibility of this method has been challenged in some studies. Our aim was to determine the intra-individual reproducibility of HRV parameters in short-term recordings obtained in supine and orthostatic positions. Electrocardiographic (ECG) recordings were obtained from 30 healthy subjects (20-49 years, 14 men) using a digital apparatus (sampling ratio $=250 \mathrm{~Hz}$ ). ECG was recorded for $10 \mathrm{~min}$ in the supine position and for $10 \mathrm{~min}$ in the orthostatic position. The procedure was repeated 2-3 $\mathrm{h}$ later. Time and frequency domain analyses were performed. Frequency domain included low (LF, 0.04-0.15 Hz) and high frequency $(\mathrm{HF}, 0.15-0.4 \mathrm{~Hz}$ ) bands. Power spectral analysis was performed by the autoregressive method and model order was set at 16. Intra-subject agreement was assessed by linear regression analysis, test of difference in variances and limits of agreement. Most HRV measures (pNN50, RMSSD, LF, HF, and LF/HF ratio) were reproducible independent of body position. Better correlation indexes $(r>0.6)$ were obtained in the orthostatic position. Bland-Altman plots revealed that most values were inside the agreement limits, indicating concordance between measures. Only SDNN and NNv in the supine position were not reproducible. Our results showed reproducibility of HRV parameters when recorded in the same individual with a short time between two exams. The increased sympathetic activity occurring in the orthostatic position probably facilitates reproducibility of the HRV indexes.
\end{abstract}

Key words: Heart rate variability; Power spectral analysis; Reproducibility; Autonomic nervous system

\section{Introduction}

Heart rate variability (HRV) is a noninvasive tool used to assess autonomic modulation of cardiac activity and either temporal or spectral measures have been used to evaluate several parameters of cardiovascular health (1). Changes of neural heart modulation occur in several systemic diseases, such as diabetes (2-5), hypertension (6), heart failure $(7,8)$, and coronary heart disease $(9,10)$. In such situations, HRV analysis can be used to monitor long-term changes of cardiac regulation by the autonomic nervous system. Reduced HRV is a remarkable predictor of cardiac events including sudden death in subjects with coronary artery disease, myocardial infarction and heart failure $(11,12)$.

However, the use of HRV to determine the neural autonomic balance directed at the heart is controversial because modulation of heart beats is strongly influenced by environmental conditions, mental stress, and body position (4). Therefore, determination of HRV reproducibility is a key question in longitudinal studies or in clinical conditions, where the same data are recorded from a single individual at different times. Although several studies have found an adequate reproducibility of HRV both in the time and in the frequency domain analyses (13-22), others have not made this observation $(22,23)$. However, reproducibility of the different components of spectral HRV obtained from short-term records is still under discussion $(24,25)$.

Conflicting reports may be due to different conditions during signal recording or different analytical procedures. Thus, studies carried out on healthy individuals have shown good reproducibility of temporal parameters when

Correspondence: J.G. Mill, Av. Marechal Campos, 1468, Maruípe, 29042-751 Vitória, ES, Brasil. Fax: +55-27-3335-7330.

E-mail: josegmill@gmail.com

Received December 21, 2009. Accepted September 8, 2010. Available online October 8, 2010. Published October $18,2010$. 
records were obtained within short ( $<24 \mathrm{~h})$ and long ( $>24 \mathrm{~h}$ ) recording periods $(18,20,21,26)$. However, even with long recordings, reproducibility has been questioned in relation to spectral parameters (22,27-29). In short-term recordings (5-10 $\mathrm{min}$ ) reproducibility increases when breathing periodicity is externally controlled (18). However, this maneuver may interfere with the high frequency component of HRV. Previous studies on intra-indivudual reproducibility of HRV have been conducted with subjects in the classical supine position $(17,22,26)$. Since this exam sometimes is also carried out during postural maneuver (tilt-test for example), we compared the reproducibility of temporal and spectral HRV indexes of a group of healthy individuals in the supine and orthostatic positions using short-term recording periods under spontaneous breathing.

\section{Material and Methods}

\section{Electrocardiogram acquisition}

Thirty apparently healthy volunteers (14 men and 16 women, $20-49$ years, mean age $=28$ years) taking no medication participated in this study. The study was approved by the Ethics Committee of the Center of Health Sciences, UFES (No. 041/2006) and all subjects gave written informed consent to participate. Electrocardiogram (ECG) acquisition was carried out at a sampling ratio of $250 \mathrm{~Hz}$ with a digital electrocardiograph (Micromed, Brazil). Wincardio (4.4a) software was used to automatically generate the R-R interval series from a selected ECG lead (usually D2). Incorrect detections or ectopic beats were removed manually. The ECG was always obtained in the morning (8:00 to 12:00 h) after a 12- to 16-h fasting period with participants breathing spontaneously in a silent room with controlled temperature $\left(21-24^{\circ} \mathrm{C}\right)$. The first 10 -min record was obtained after a 5 -min rest in the supine position. Then, the subject was asked to stand up to obtain another 11- to 12-min ECG recording in the orthostatic position. The first minute of the recording was discarded to avoid acute interference due to the postural maneuver. The same ECG recording protocol was repeated for each subject $2 \mathrm{~h}$ after the first measurement. No consumption of food, caffeine or cigarettes was allowed between the two recordings.

\section{HRV analyses}

HRV was measured in the time and frequency domains in the two positions. Time domain analyses included mean heart rate $(\mathrm{HR})$, mean normal-to-normal (NN) interval duration (NNi), $\mathrm{NN}$ variance $(\mathrm{NNv})$, standard deviation of all $\mathrm{NN}$ intervals (SDNN), the proportion derived by dividing the number of pairs of adjacent $N N$ intervals differing by more than $50 \mathrm{~ms}$ in the entire recording (NN50) by the total number of all NN intervals (pNN50) and finally the square root of the mean of the sum of the squares of differences between adjacent $\mathrm{NN}$ intervals (RMSSD). NN intervals were considered to be valid between 0.6 and $1.6 \mathrm{~s}$ in the supine position and between 0.4 and $1.4 \mathrm{~s}$ in the orthostatic position. Data of one subject in the supine position and of two other subjects in the orthostatic position were excluded from analysis because more than $30 \%$ of $\mathrm{R}-\mathrm{R}$ intervals were out of this range. Frequency domain HRV analyses included low frequency (LF, 0.04-0.15 Hz), and high frequency $(\mathrm{HF}, 0.15-0.4 \mathrm{~Hz}$ ) bands (1). Power spectral analysis was performed by the autoregressive method and model order was set at 16 (30). Matlab-customized software developed in our laboratory was used for temporal and spectral analysis (available at ftp.ifes.edu.br/cursos/ engenhariaeletrica/rodrigo/pesquisa/vfc).

\section{Statistical analysis}

Data are reported as means \pm SEM. Data normality was tested by the Shapiro-Wilk test and variance homogeneity by the Levene test. When a variable was not normally distributed the following transformations were tested: inverse, square root, logarithm (Ln), cubic and inverse of square root. Intrasubject agreement between the first and second measurement in the same position was assessed by linear regression analysis using Pearson's product moment correlation coefficient ( $r$ ), Pitman's test of difference in variance, and Bland-Altman plot. In this last test, the difference between two measurements and the mean and standard deviation were calculated. If the values of the differences are normally distributed, it is expected that $95 \%$ of these values will be between the mean \pm 2 standard deviations. This interval is called the "agreement limit". If this interval presents a wide range of variation, it is supposed that there is no concordance between the two measurements and the reproducibility hypothesis is rejected. A visual inspection of the graph shows data dispersion. If there is concordance between two measures, the different values scatter along the zero line. Comparisons of means between the supine and orthostatic positions were performed by the two-tailed unpaired Student $t$-test or by the Wilcoxon rank-sum test. Statistical significance was set at $P<0.05$. Analysis was carried out with the STATA/SE 10.1 software.

\section{Results}

\section{Time and frequency domain analysis}

Mean values of the time and frequency domains of HRV parameters evaluated in the subjects are shown in Table 1. In the supine position, the mean values of measures 1 and 2 were similar, except for $\mathrm{HR}$ and $\mathrm{NNi}$. In the orthostatic position all variables were similar in the two acquisition periods. It is noteworthy that HRV parameters showed large differences when supine and orthostatic positions were compared. Parameters measured in the orthostatic position were almost the same during the first and second acquisition periods. Parallel to the HR increase in the orthostatic position, there were statistically significant reductions in all temporal parameters of HRV. A significant increase of the 
LF component was observed $(49 \pm 3.8$ vs $79 \pm 3.1 \mathrm{nu}$; $\mathrm{P}<$ 0.01 , first period) as HF decreased in the same proportion $(47 \pm 3.9$ vs $16 \pm 2.2 \mathrm{nu} ; \mathrm{P}<0.01)$. As a consequence, a large increase in the LF/HF ratio (from $1.99 \pm 0.69$ to 13.33 $\pm 3.51 ; \mathrm{P}<0.01$ ) was observed.

\section{Linear regression analysis}

Linear regression analysis was carried out on crude or transformed normalized variables. Table 2 shows significant linear associations between the first and the second acquisition periods in the two positions. All measures for time- and frequency-domain analysis presented a high degree of intra-individual reproducibility. Association degree assessed by the Pearson correlation coefficient was higher than 0.80 for 11 of 18 parameters.

\section{Bland-Altman test and Pitman test of difference in variances}

Bland-Altman plots revealed that almost all data were inside agreement limits, except for the SDNN and NNv variables in the supine position where three individuals were out of the agreement limits (Figure 1). Figure 1 also shows plots of some measures in the time (SDNN) and frequency domains (LF, HF, and LF/HF) in the supine and the orthostatic positions. There was also agreement between the first and second measures for $\mathrm{NNi}$, NNv, pNN50, and RMSSD (data not shown). Similar to the time domain parameters, the spectral values and the LF/HF ratio also showed good reproducibility according to Bland-Altman analysis. To further investigate intra-individual reproducibility, the equivalency of variances was tested in acquisition periods 1 and 2 and the Pitman test confirmed a significant difference $(P=0.022)$ in the variability between measurements 1 and 2 only for SDNN and NNv parameters in the supine position. For all other variables, no significant differences $(P>0.05)$ were demonstrable, indicating that the measurements were reproducible.

\section{Discussion}

There is a growing interest in the use of HRV to assess the sympathetic/parasympathetic control of heart beats. Since it is a noninvasive and inexpensive method, several recordings can be easily obtained as a function of time in the same individual to evaluate the efficacy of therapeutic interventions. Therefore, some studies have been specifically designed to evaluate the reproducibility of the measurements since autonomic balance is subjected to a large number of central and peripheral control systems, all of them potentially affecting HR control $(17-22,25)$. Some studies have shown good reproducibility in HRV analysis $(17,20,21)$, while others have reported the opposite $(22,27)$. Studies on this subject have shown that reproducibility is

Table 1. Values of heart rate variability parameters.

\begin{tabular}{lccccc}
\hline & \multicolumn{2}{c}{ Supine position $(\mathrm{N}=29)$} & & \multicolumn{2}{c}{ Orthostatic position (N=28) } \\
\cline { 2 - 3 } \cline { 6 - 6 } & Recording 1 & Recording 2 & & Recording 1 & Recording 2 \\
\hline HR (bpm) & $65 \pm 1.9$ & $63 \pm 1.7^{+}$ & & $84 \pm 2.2^{*}$ & $85 \pm 2.2^{*}$ \\
NNi (ms) & $936 \pm 27.6$ & $962 \pm 25.9^{+}$ & & $727 \pm 19.8^{*}$ & $718 \pm 18.4^{*}$ \\
NNv (ms) & $5781 \pm 9945$ & $6526 \pm 1007$ & & $4449 \pm 1184$ & $3790 \pm 576$ \\
SDNN (ms) & $68 \pm 6.1$ & $74 \pm 5.9$ & & $60 \pm 5.5$ & $57 \pm 4.0$ \\
pNN50 (\%) & $28 \pm 3.9$ & $28 \pm 3.6$ & & $6 \pm 1.5^{*}$ & $5 \pm 1.1^{*}$ \\
RMSSD (ms) & $66 \pm 7.6$ & $66 \pm 6.7$ & & $32 \pm 4.6^{*}$ & $33 \pm 5.6^{*}$ \\
LF (nu) & $49 \pm 3.8$ & $51 \pm 4.0$ & & $79 \pm 3.1^{*}$ & $77 \pm 4.0^{*}$ \\
HF (nu) & $47 \pm 3.9$ & $43 \pm 4.1$ & & $16 \pm 2.2^{*}$ & $18 \pm 3.4^{*}$ \\
LF/HF & $1.99 \pm 0.69$ & $1.88 \pm 0.30$ & & $13.33 \pm 3.51^{*}$ & $10.77 \pm 2.46^{*}$ \\
\hline
\end{tabular}

$\mathrm{HR}=$ heart rate; $\mathrm{NNi}=$ normal-to-normal interval duration; $\mathrm{NNv}=\mathrm{NN}$ variance; SDNN = standard deviation of all NN intervals; pNN50 = total number of all NN intervals; RMSSD = sum of the squares of differences between adjacent NN intervals; LF = low frequency; HF = high frequency; nu = normalized units. ${ }^{+} \mathrm{P}<0.05$, recording 2 compared to recording 1 in the supine or orthostatic position. ${ }^{*} \mathrm{P}<0.05$, orthostatic compared to supine position. Comparisons were performed between recordings 1 and 2 by the two-tailed Student $t$-test for paired samples or by the Wilcoxon matched-pairs signed-rank test. Comparisons between the supine and orthostatic positions were carried out using the unpaired two-tailed Student $t$-test or Wilcoxon rank-sum test.

Table 2. Correlation of heart rate variability parameters.

\begin{tabular}{|c|c|c|c|c|}
\hline \multirow[t]{2}{*}{ Variable } & \multicolumn{2}{|c|}{ Supine position ( $\mathrm{N}=29)$} & \multicolumn{2}{|c|}{ Orthostatic position $(\mathrm{N}=28)$} \\
\hline & Transformation & $(r)$ & Transformation & $(r)$ \\
\hline HR1 x HR2 & None & 0.9269 & None & 0.9130 \\
\hline NNi1 x Nni2 & None & 0.9240 & None & 0.8865 \\
\hline NNv1 x NNv2 & $1 / \sqrt{ }$ variable & 0.8529 & $1 / \sqrt{ }$ variable & 0.7476 \\
\hline SDNN1 x SDNN2 & 1/variable & 0.8529 & 1/variable & 0.7476 \\
\hline pNN501 x pNN502 & Vvariable & 0.8255 & Ln variable & 0.8559 \\
\hline RMSSD1 x RMSSD2 & $1 / \sqrt{ }$ variable & 0.8432 & $1 / \sqrt{ }$ variable & 0.9119 \\
\hline LF1 x LF2 & None & 0.7506 & $(\text { variable })^{3}$ & 0.8919 \\
\hline HF1 x HF2 & None & 0.7400 & Ln variable & 0.7941 \\
\hline LF/HF1 x LF/HF2 & Vvariable & 0.6654 & Ln variable & 0.7772 \\
\hline
\end{tabular}

1 = first recording; 2 = second recording; $N=$ number of individuals. $(r)=$ Pearson's correlation coefficient. For other abbreviations, see Table 1. P values for all correlation coefficients were $<0.0001$. 

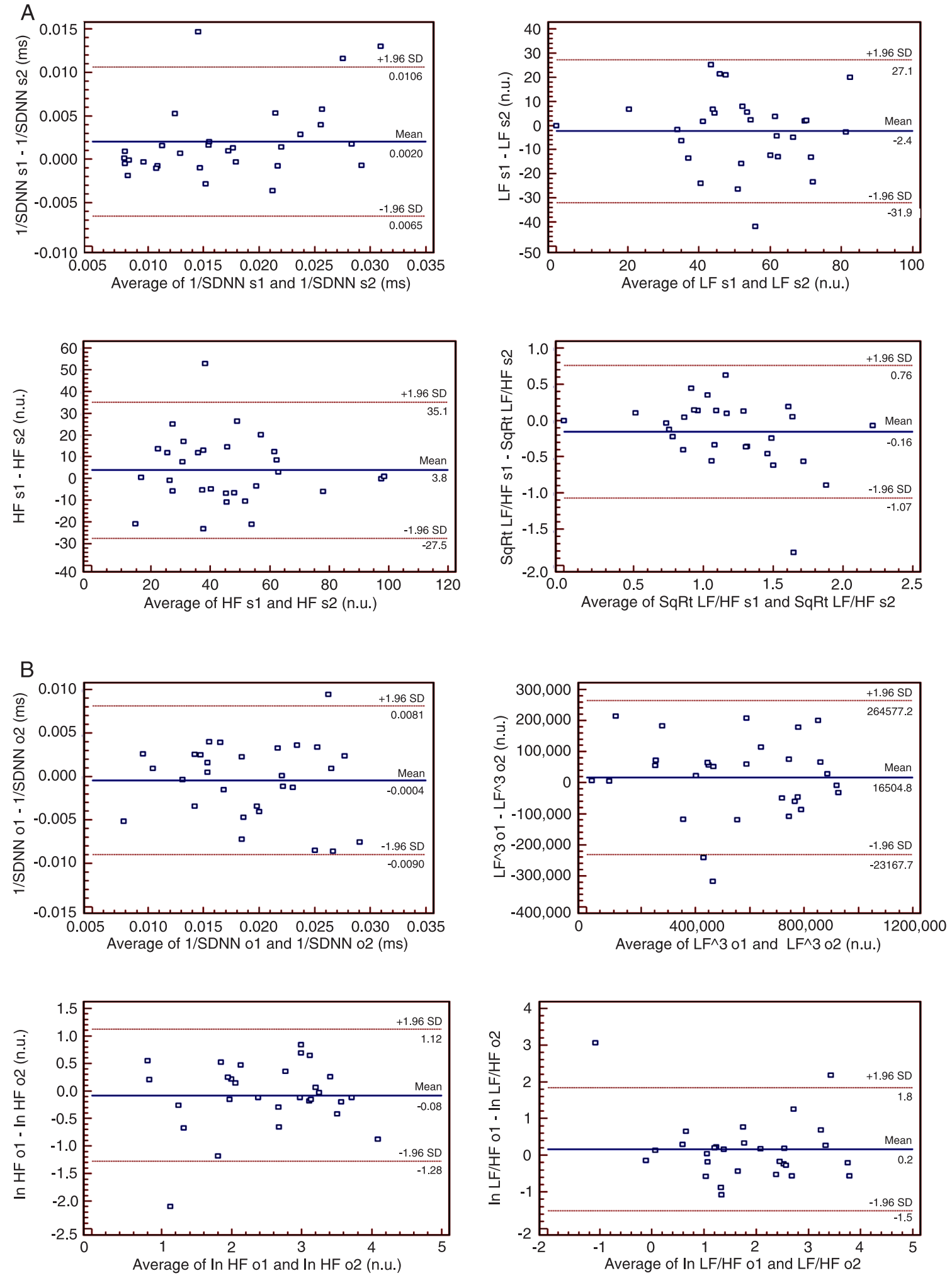

Figure 1. Bland-Altman plots of some heart rate variability parameters assessed in the supine $(A)$ and orthostatic (B) positions. n.u. = normalized units; s1 and s2 = first and second measurements in supine position; 01 and $02=$ first and second measurements in orthostatic position. For abbreviations, see legend to Table 1. 
generally high for long-time recordings (24-h Holter) and for the time-domain parameters obtained in short-time recordings (5-10 min). Intra-individual reproducibility of spectral parameters obtained in short-time recordings, however, is still scarce and its results heterogeneous (17). Therefore, we investigated this parameter in two exams conducted on healthy volunteers under identical conditions during the same morning period. Additionally, we also tested reproducibility in the orthostatic position, a maneuver designed to enhance the sympathetic drive. In general, our data showed high reproducibility both in the time and frequency domain analysis, mainly when the ECG recording was obtained in the orthostatic position.

Reproducibility of HRV measures depends on several factors, including local conditions (temperature, noise), mental stress, spontaneous or paced breathing, and time interval between measurement $(17,18,25)$. Therefore, recordings were obtained under rigorous conditions to reduce factors that might impair reproducibility. Intraindividual variability can occur due to the intrinsic instability of HRV parameters consequent to changes of mood, alertness, and mental activity. In clinical conditions these elements, indeed, are very difficult to control $(7,17)$. Therefore, to reduce the influence of these factors, the ECG was obtained in a silent room, at a comfortable temperature and volunteers were stimulated to stay awake and mentally relaxed throughout the recording periods. Some discomfort may occur during orthostatic recording, mainly in cases where blood pressure cannot be maintained at adequate levels. In our study, however, the exam carried out on healthy subjects was well tolerated.

A notable influence of the postural change maneuver on HRV parameters was observed (Table 1). When subjects changed from the supine to the orthostatic position there were modifications in all parameters in the time and frequency domains due to the increase of sympathetic activity and the decrease of parasympathetic activity. The first minute of the recording in the orthostatic position was removed from the analysis to minimize the acute interference imposed by the change in body position. Of note in the temporal domain analysis was the dramatic decrease of pNN50, which is closely related to the parasympathetic tone (18). In spectral analysis, since LF and HF change in opposite directions in the postural maneuver, a large increase of the LF/HF ratio was observed, indicating a strong dominance of the sympathetic drive in heart beat control. As stated before, our data indicate better reproducibility indexes in the orthostatic position than in the supine position. In contrast to the supine position, even SDNN was reproducible when subjects were standing. Our results agree with previous findings obtained in healthy old women (31), which also demonstrated better reliability of measurements obtained in the upright position. These data suggest that HRV measures in the supine position are more sensitive to variations of the sympathetic activity caused by external factors such as mood, stress, sleep, and anxiety. The increase of the sympathetic drive in the orthostatic position may reduce the influence of such external factors, improving intra-individual stability of HRV indexes. Therefore, our results supply new evidence for the use of the orthostatic position in data collection when the objective is to reduce external influences and to increase the reliability of HRV indexes in longitudinal studies. However, additional studies are necessary to determine if these findings are demonstrable in subjects with diseases that cause disturbances of the autonomic nervous system.

Unlike other studies $(22,27)$, our results indicate that intra-individual measurements can show good reproducibility, even for short-time recordings in the supine position if external factors that alter autonomic balance are well controlled. Thus, studies questioning reproducibility use different ECG recording sequences. For example, Lord et al. (22) evaluated reproducibility of measurements performed at different times of day: in the morning (between 8:00 and 9:00 h), in the early afternoon (between 12:00 and 13:00 h) and in the late afternoon (between 15:00 and 16:00 h). Moreover, the second recording was repeated 1 and 2 weeks after the first. The results suggested day-to-day differences and some significant differences were also found between measurements at different times of the same day. In another study (18) on healthy subjects, HRV was assessed three times. The first two measurements were separated by 2 weeks and the third evaluation was obtained $210 \pm 25$ days later. ECG signals were analyzed in the time domain using 24-h or 10-min recordings. Frequency domain analyses were carried out from short-period recordings. In time domain analyses, there were no differences in the values of the parameters obtained during three 24-h ECG monitoring periods. However, the reproducibility of the temporal parameters was low or fair when 10-min ECG recordings were used. In the frequency domain analyses, only total power and LF showed reproducibility. Finally, Højgaard et al. (27) evaluated reproducibility using a protocol consisting of $1 \mathrm{~h}$ of supine rest recordings in 14 healthy individuals on three different days for the assessment of day-to-day and short-term reproducibility. In that study, there was only moderate-to-poor reproducibility of the spectral parameters of HRV (coefficient of variation range 18-36\%). Therefore, the poor reproducibility found in these studies seems to occur primarily due to the long time interval between measurements. According to our data, shorter time intervals are better to obtain agreement between measurements, and the ECG acquisition in the orthostatic position can improve reproducibility along time.

Finally, some issues need to be addressed. Although the HRV guidelines (1) recommend the use of 5-min records, because at least $1 \mathrm{~min}$ is needed to assess HF components and about 2 min to obtain the LF component, we used a longer recording of $10 \mathrm{~min}$. The main reason for this choice is the fact that the data collected may contain 
artifacts generating abnormal $\mathrm{R}-\mathrm{R}$ intervals, which must be excluded before analysis. Thus, to ensure an acceptable recording of $5 \mathrm{~min}$, a longer recording was required. Our study investigated the reproducibility of the HRV parameters in both the time and frequency domains, and short-term recordings (5-10 $\mathrm{min}$ ) are more appropriate for frequency domain analyses.

We have shown that there was a high level of reproducibility in time and frequency domain parameters of HRV, especially when the ECG was recorded in the orthostatic position. Compared with previous studies, our data indicate that a small time interval between two recordings seems to be the main factor influencing the reproducibility of HRV measurements even at free respiration. Considering that reproducibility was better when the ECG was recorded in the orthostatic position, the results of our study also suggest that this position should be used preferentially, instead of the supine position, in situations in which the same exam should be obtained at different times in the same individual. It is likely that the increased sympathetic activity occurring in the orthostatic position facilitates reproducibility of the main HRV indexes. However, as the autonomic modulation depends on body position, the use of the supine position is preferred when the objective is to assess the subject in situations with a predominance of vagal modulation of the heart beat, and the orthostatic position is preferred when the aim is to evaluate the subject in situations with a predominance of sympathetic activity.

\section{References}

1. Task Force of the European Society of Cardiology and the North American Society of Pacing and Electrophysiology. Heart rate variability. Standards of measurement, physiological interpretation, and clinical use. Eur Heart $J$ 1996; 17: 354-381.

2. Burger AJ, Charlamb M, Weinrauch LA, D'Elia JA. Shortand long-term reproducibility of heart rate variability in patients with long-standing type I diabetes mellitus. Am J Cardiol 1997; 80: 1198-1202.

3. Nolan J, Flapan AD, Goodfield NE, Prescott RJ, Bloomfield $\mathrm{P}$, Neilson JM, et al. Measurement of parasympathetic activity from 24-hour ambulatory electrocardiograms and its reproducibility and sensitivity in normal subjects, patients with symptomatic myocardial ischemia, and patients with diabetes mellitus. Am J Cardiol 1996; 77: 154-158.

4. Ewing DJ, Neilson JM, Shapiro CM, Stewart JA, Reid W. Twenty four hour heart rate variability: effects of posture, sleep, and time of day in healthy controls and comparison with bedside tests of autonomic function in diabetic patients. Br Heart J 1991; 65: 239-244.

5. Malpas SC, Maling TJ. Heart-rate variability and cardiac autonomic function in diabetes. Diabetes 1990; 39: 1177 1181.

6. Konrady AO, Rudomanov OG, Yacovleva OI, Shlyakhto EV. Power spectral components of heart rate variability in different types of cardiac remodelling in hypertensive patients. Med Sci Monit 2001; 7: 58-63.

7. Stein PK, Rich MW, Rottman JN, Kleiger RE. Stability of index of heart rate variability in patients with congestive heart failure. Am Heart J 1995; 129: 975-981.

8. Scalvini S, Volterrani M, Zanelli E, Pagani M, Mazzuero G, Coats AJ, et al. Is heart rate variability a reliable method to assess autonomic modulation in left ventricular dysfunction and heart failure? Assessment of autonomic modulation with heart rate variability. Int J Cardiol 1998; 67: 9-17.

9. Bigger JT Jr, Fleiss JL, Steinman RC, Rolnitzky LM, Schneider WJ, Stein PK. RR variability in healthy, middle-aged persons compared with patients with chronic coronary heart disease or recent acute myocardial infarction. Circulation 1995; 91: 1936-1943.

10. Weber F, Schneider H, von Arnim T, Urbaszek W. Heart rate variability and ischaemia in patients with coronary heart disease and stable angina pectoris; influence of drug therapy and prognostic value. TIBBS Investigators Group. Total Ischemic Burden Bisoprolol Study. Eur Heart J 1999; 20: 38-50.

11. van Boven AJ, Jukema JW, Haaksma J, Zwinderman AH, Crijns HJ, Lie KI. Depressed heart rate variability is associated with events in patients with stable coronary artery disease and preserved left ventricular function. REGRESS Study Group. Am Heart J 1998; 135: 571-576.

12. Nolan J, Batin PD, Andrews R, Lindsay SJ, Brooksby P, Mullen $\mathrm{M}$, et al. Prospective study of heart rate variability and mortality in chronic heart failure: results of the United Kingdom heart failure evaluation and assessment of risk trial (UK-heart). Circulation 1998; 98: 1510-1516.

13. Van Hoogenhuyze D, Weinstein N, Martin GJ, Weiss JS, Schaad JW, Sahyouni XN, et al. Reproducibility and relation to mean heart rate of heart rate variability in normal subjects and in patients with congestive heart failure secondary to coronary artery disease. Am J Cardiol 1991; 68: 16681676.

14. Kamalesh M, Burger AJ, Kumar S, Nesto R. Reproducibility of time and frequency domain analysis of heart rate variability in patients with chronic stable angina. Pacing Clin Electrophysiol 1995; 18: 1991-1994.

15. Kautzner J, Hnatkova K, Staunton A, Camm AJ, Malik M. Day-to-day reproducibility of time-domain measures of heart rate variability in survivors of acute myocardial infarction. Am J Cardiol 1995; 76: 309-312.

16. Pardo Y, Merz CN, Paul-Labrador M, Velasquez I, Gottdiener JS, Kop WJ, et al. Heart rate variability reproducibility and stability using commercially available equipment in coronary artery disease with daily life myocardial ischemia. $A m \mathrm{~J}$ Cardiol 1996; 78: 866-870.

17. Pinna GD, Maestri R, Torunski A, Danilowicz-Szymanowicz L, Szwoch M, La Rovere MT, et al. Heart rate variability measures: a fresh look at reliability. Clin Sci 2007; 113: 131140.

18. Pitzalis MV, Mastropasqua F, Massari F, Forleo C, Di Maggio M, Passantino A, et al. Short- and long-term reproducibility of time and frequency domain heart rate variability mea- 
surements in normal subjects. Cardiovasc Res 1996; 32: 226-233.

19. Parati G, Omboni S, Villani A, Glavina F, Castiglioni P, Di Rienzo M, et al. Reproducibility of beat-by-beat blood pressure and heart rate variability. Blood Press Monit 2001; 6: 217-220.

20. Marks BL, Lightfoot JT. Reproducibility of resting heart rate variability with short sampling periods. Can J Appl Physiol 1999; 24: 337-348.

21. Cloarec-Blanchard L, Funck-Brentano C, Lipski M, Jaillon $P$, Macquin-Mavier I. Repeatability of spectral components of short-term blood pressure and heart rate variability during acute sympathetic activation in healthy young male subjects. Clin Sci 1997; 93: 21-28.

22. Lord SW, Senior RR, Das M, Whittam AM, Murray A, McComb JM. Low-frequency heart rate variability: reproducibility in cardiac transplant recipients and normal subjects. Clin Sci 2001; 100: 43-46.

23. Ponikowski P, Piepoli M, Amadi AA, Chua TP, Harrington D, Volterrani $M$, et al. Reproducibility of heart rate variability measures in patients with chronic heart failure. Clin Sci 1996; 91: 391-398.

24. Parati G, Mancia G, Di Rienzo M, Castiglioni P. Point: cardiovascular variability is/is not an index of autonomic control of circulation. J Appl Physiol 2006; 101: 676-678.

25. Sandercock GR, Bromley PD, Brodie DA. The reliability of short-term measurements of heart rate variability. Int $J$ Cardiol 2005; 103: 238-247.

26. Kleiger RE, Bigger JT, Bosner MS, Chung MK, Cook JR, Rolnitzky LM, et al. Stability over time of variables measuring heart rate variability in normal subjects. Am J Cardiol 1991; 68: 626-630.

27. Hojgaard MV, Holstein-Rathlou NH, Agner E, Kanters JK. Reproducibility of heart rate variability, blood pressure variability and baroreceptor sensitivity during rest and head-up tilt. Blood Press Monit 2005; 10: 19-24.

28. Piepoli M, Radaelli A, Ponikowski P, Adamopoulos S, Bemardi L, Sleight $P$, et al. Reproducibility of heart rate variability indices during exercise stress testing and inotrope infusion in chronic heart failure patients. Clin Sci 1996; 91 (Suppl): 87-88.

29. Salo TM, Voipio-Pulkki LM, Jalonen JO, Helenius H, Viikari JS, Kantola I. Reproducibility of abnormal heart rate variability indices: the case of hypertensive sleep apnoea syndrome. Clin Physiol 1999; 19: 258-268.

30. Boardman A, Schlindwein FS, Rocha AP, Leite A. A study on the optimum order of autoregressive models for heart rate variability. Physiol Meas 2002; 23: 325-336.

31. Reland S, Ville NS, Wong S, Carrault G, Carre F. Reliability of heart rate variability in healthy older women at rest and during orthostatic testing. Aging Clin Exp Res 2005; 17: 316321. 